\title{
Efecto in vitro de controladores biológicos sobre Colletotrichum spp. y Botrytis spp.
}

\section{In vitro effect of biological controllers on Colletotrichum spp. and Botrytis spp.}

\author{
Malluri Goñas Goñas ${ }^{1 *}$, Leidy Gheraldinne Bobadilla Rivera ${ }^{1}$, Jesús Rascón Barrios ${ }^{1}$ y Nora Yessenia Vera Obando
}

\section{RESUMEN}

La presente investigación, realizada en el Laboratorio de Investigación de Entomología y Fitopatología de la Universidad Nacional Toribio Rodríguez de Mendoza de Amazonas, tuvo el objetivo de evaluar el efecto in vitro de cuatro controladores biológicos (Trichoderma harzianum, T. viride, T. asperellum y Clonostachys rosea) frente a los hongos patógenos Colletotrichum spp. y Botrytis spp. Se utilizó la metodología de cultivos duales, que consiste en enfrentar el enemigo biológico en un extremo de una placa Petri y el hongo patógeno en el otro. Se establecieron seis tratamientos que incluyeron un testigo absoluto y un testigo químico (propineb $1000 \mathrm{ppm}$ ) por patógeno, con cinco repeticiones por tratamiento. Se logró el aislamiento de los fitopatógenos Colletotrichum spp., agente causal de la antracnosis del mango, y Botrytis spp., agente causal de la rancha en azucenas. Al comparar el crecimiento radial cada 24 horas, se encontró que 96 horas después del enfrentamiento, las cepas de Trichoderma inhibieron el crecimiento radial de los patógenos, diferenciándose significativamente del testigo absoluto. Al evaluar el porcentaje de inhibición del crecimiento radial (PICR) en Botrytis spp., las cepas de Trichoderma spp. presentaron mayor PICR, sin embargo, no presentaron diferencias significativas con el testigo químico. En Colletotricum spp., las cepas de Trichoderma spp. presentaron mayor PICR, y además diferencias significativas estadísticas con el testigo químico. Finalmente, cabe destacar que la cepa de $C$. rosea no inhibió el crecimiento radial de los patógenos, mostrando los PICR más bajos.

Palabras clave: Antracnosis, controlador biológico, crecimiento radial, azucena, mango.

\begin{abstract}
The present research was carried out in the Laboratory of Entomology and Phytopathology Research of the National University Toribio Rodriguez de Mendoza of Amazonas, and had the objective to evaluate the in vitro effect of four biological controllers (Trichoderma harzianum, T. viride, T. asperellum and Clonostachys rosea) against pathogenic fungi Colletotrichum spp. and Botrytis spp. We used the dual crops methodology, which consisted of facing the biological enemy at one end of a Petri dish and the pathogenic fungus at the other. Six treatments were established that included an absolute control and a chemical control (propineb 1000ppm) per pathogen, with five replicates per treatment. Isolation of phytopathogens Colletotrichum spp., causal agent of anthracnose of mango, and Botrytis spp., causal agent of the late blight in lilies. When comparing the radial growth in 24 hours, it was found that 96 hours after the confrontation, the Trichoderma strains inhibited the radial growth of the pathogens, differing significantly from the absolute control. When evaluating the percentage of inhibition of radial growth (RCIP) in Botrytis spp., the strains of Trichoderma spp. presented higher RCIPs, however, they did not present significant differences with the chemical control. In contrast, in Colletotricum spp., strains of Trichoderma spp. presented higher RCIP and also significant statistical differences with the chemical control. Finally, it should be noted that the strain of C. rosea did not inhibit the radial growth of the pathogens, showing the lowest RCIPs.
\end{abstract}

Keywords: Anthracnose, biological controller, radial growth, lily, mango.

\footnotetext{
${ }^{1}$ Universidad Nacional Toribio Rodríguez de Mendoza, Chachapoyas, Amazonas, Perú

*Autor de correspondencia. E-mail: malluri.gonas@untrm.edu.pe
} 


\section{INTRODUCCIÓN}

Hay un interés muy grande en identificar la capacidad de los patógenos para evolucionar y adaptarse a nuevos hábitats, especialmente en el caso de enfermedades emergentes devastadoras (Archie et al., 2009). En este sentido, los hongos están entre los patógenos más devastadores de las plantas causando, principalmente, importantes pérdidas en la agricultura, (Anderson et al., 2004)

Colletotrichum spp. es un hongo cosmopolita reconocido como causante de daños a los órganos de muchos cultivos agrícolas, forestales y especies ornamentales. El control de las enfermedades causadas por especies de Colletotrichum depende del uso de semillas sanas, semillas tratadas con agua caliente, selección de variedades resistentes, rotación de cultivos, gestión de los productos durante y después de la cosecha, y tratamiento del embalaje y de los contenedores donde se trasladan los frutos (Hernández et al., 2013)

La susceptibilidad que muestran los frutos de mango a las enfermedades, la cual se incrementa después de la cosecha, en post cosecha, es un factor limitante para las exportaciones y comercialización, debido a la pudrición que ocasiona el patógeno en el fruto, y la consiguiente pérdida de su valor comercial, lo que puede ocasionar pérdidas que fluctúan entre el 30\% y el $60 \%$ del total de la producción (Santander, 2012).

Por otro lado, Flores et al. (2015) mencionan que Botrytis spp, es el patógeno que causa la enfermedad del moho gris en una amplia variedad de huéspedes, y es un grave problema económico en cultivos como uvas, fresas, frambuesas, lechugas, pepinos, habas, tomates, frijoles, incluso flores y plantas forestales producidas en contenedores y en algunos casos en algunos casos causan pérdidas superiores al 50\%.

Otra especie ampliamente afectada por el género Botrytis es la azucena (Lillium spp.), y es que tanto su producción como su propio rendimiento pueden estar afectados por el ataque de hongos de este género (Leyva-Mir et al., 2009). Actualmente se considera que el control biológico de patógenos de plantas, con el uso de microorganismos benignos o beneficiosos como agentes antagonistas es un componente importante del manejo integrado de plagas en cultivos agrícolas (Ryu et al., 2014). También es una práctica muy importante para el manejo de plagas, al utilizar organismos vivos para reducir y mantener la abundancia poblacional de una plaga por debajo de los niveles de daño económico, ya que su valor recae en que puede resultar en un control eficiente de una plaga tanto a mediano como a largo plazo, compatible con un bajo riesgo ambiental y una producción sustentable (Farooq et al., 2011). En este sentido, resulta fundamental para los programas de control biológico considerar la ecología, biología y comportamiento de los enemigos naturales de la plaga y de la plaga misma, además de aquellos factores que podrían ser causantes de cambios poblacionales (Fischbein, 2012).

Los mecanismos de biocontrol atribuidos a Trichoderma spp. son: micoparasitismo, competencia por los nutrientes y antibiosis, siendo el micoparasitismo el principal mecanismo de acción de este hongo. Este biocontrolador cubre al hongo, ataca y penetra en sus células causándole un daño extensivo alterando y degradando la pared celular, causa retracción de la membrana plasmática y desorganización del citoplasma (Guédeza et al., 2009), a esto se suma la secreción de enzimas y la producción de compuestos inhibidores (Infante et al., 2009)

Además se conoce que Trichoderma presenta otros mecanismos, cuya acción biorreguladora es de forma indirecta. Entre estos se pueden mencionar los que elicitan o inducen mecanismos de defensa fisiológicos y bioquímicos como es la activación en la planta de compuestos relacionados con la resistencia (Inducción de Resistencia), con la detoxificación de toxinas excretadas por patógenos y la desactivación de enzimas de estos durante el proceso de infección (Infante et al., 2009)

El objetivo del presente trabajo fue evaluar el efecto in vitro de controladores biológicos (Trichoderma harzianum, Trichoderma viride, Trichoderma asperellum y Clonostachys rosea) sobre el agente causal de la antracnosis del mango (Colletotricum spp) y la rancha 
en azucena (Botrytis spp.), en Chachapoyas (Departamento de Amazonas).

\section{MATERIALES Y MÉTODOS}

\section{Ubicación de la investigación}

El estudio se llevó acabo en el Laboratorio de Investigación de Etimología y Fitopatología de la Universidad Nacional Toribio Rodríguez de Mendoza de Amazonas, situado en la ciudad de Chachapoyas, capital del Departamento Amazonas.

\section{Aislamiento de fitopatógenos}

Para aislar en medio de cultivo Agar Papa Dextrosa (PDA) e identificar Colletotrichum spp. a partir de frutos de mango, y Botrytis spp. a partir de hojas de azucena, se siguió la metodología descrita por Krugg y Requejo-Valdivieso (2012), con algunas modificaciones para el desarrollo de esta investigación. Se procedió a hacer cortes en cuadrados de aproximadamente $0,5 \mathrm{~cm}$ de lado a partir del borde de la lesión que contengan tanto tejido sano como enfermo. Estos cortes fueron puestos en agua corriente durante una hora. Transcurrido este tiempo fueron tratados durante tres minutos en una solución de hipoclorito de sodio al $1 \%$. A continuación, se lavaron tres veces con agua destilada estéril para dejarlos secar en placas Petri con papel toalla estéril. Finalmente fueron colocados en placas Petri conteniendo PDA, e incubadas a $26^{\circ} \mathrm{C}$ por 5 a 7 días.

\section{Identificación de fitopatógenos}

De cada una de las colonias que se desarrollaron en las placas con PDA, se realizaron observaciones microscópicas con azul de lactofenol, y paralelamente, se sembraron en tubos conteniendo PDA, con el fin de obtener cultivos puros.

\section{Prueba de antagonismo}

Para la prueba de antagonismo se utilizó la metodología de cultivos duales utilizando placas Petri conteniendo medio de cultivo PDA, en las cuales se sembró el enemigo biológico en un extremo de la placa y el hongo fitopatógeno en el otro (aproximadamente a 7 $\mathrm{cm}$ de distancia). Se utilizaron cinco placas Petri por cada tratamiento, incubándolas a una temperatura de $25^{\circ} \mathrm{C} \pm 2^{\circ} \mathrm{C}$. Posteriormente, para la comparación del crecimiento radial de los fitopatógenos enfrentados a los biocontroladores con los testigos patógenos, se realizaron observaciones y mediciones cada 24 horas, hasta que el desarrollo fúngico de los testigos absolutos cubriera completamente la placa Petri. En esta etapa se calculó el Porcentaje de Inhibición del crecimiento radial (PICR), utilizando la fórmula:

$$
\mathrm{PICR}=\frac{\mathrm{R}_{1}-\mathrm{R}_{2}}{\mathrm{R}_{2}} \times 100
$$

Donde:

$\mathrm{R}_{1}=$ radio en cm del patógeno testigo absoluto

$\mathrm{R}_{2}=$ radio en cm del patógeno enfrentado.

\section{Diseño experimental}

Se trabajó con un Diseño Completo al Azar (DCA), utilizando cuatro cepas comerciales de controladores biológicos: Trichoderma harzianum, Trichoderma viride, Trichoderma asperellum, Clonostachys rosea, enfrentados a Colletotricum spp y Botrytis spp. Las cepas de los controladores biológicos se obtuvieron del Laboratorio de Control Biológico del Servicio Nacional de Sanidad Agraria. Los tratamientos de estudio para Colletotricum spp. fueron: T1, Colletorichum spp. vs T. harzianum; T2, Colletorichum spp. vs T. viride; T3, Colletorichum spp. vs T. asperellum; T4, Colletorichum spp. vs Clonostachis rosea; T5, Colletorichum spp. testigo químico (Propineb a 1000ppm); T6, (Colletotricum spp. Testigo absoluto). Los tratamientos de estudio para Botrytis spp. fueron: T1, Botrytis spp. vs T. harzianum; T2, Colletorichum spp. vs $T$. viride; T3, Botrytis spp. vs T.a asperellum; T4, Botrytis spp. vs C. rosea; T5, Botrytis spp. testigo químico; T6, Botrytis spp. testigo absoluto.

\section{RESULTADOS}

Aislamiento e identificación de Botrytis spp. y Colletotrichum spp.

De hojas de azucena se logró aislar e identificar a Botrytis spp. como el agente causal de la rancha en 
azucenas; seis días después de la incubación el crecimiento micelial llenó por completo la placa Petri, el color de las colonias fue de blanco a gris claro, y al observar estas colonias en el microscopio se identificaron conidióforos en forma de racimo, característico de Botrytis spp. (Figura 1).

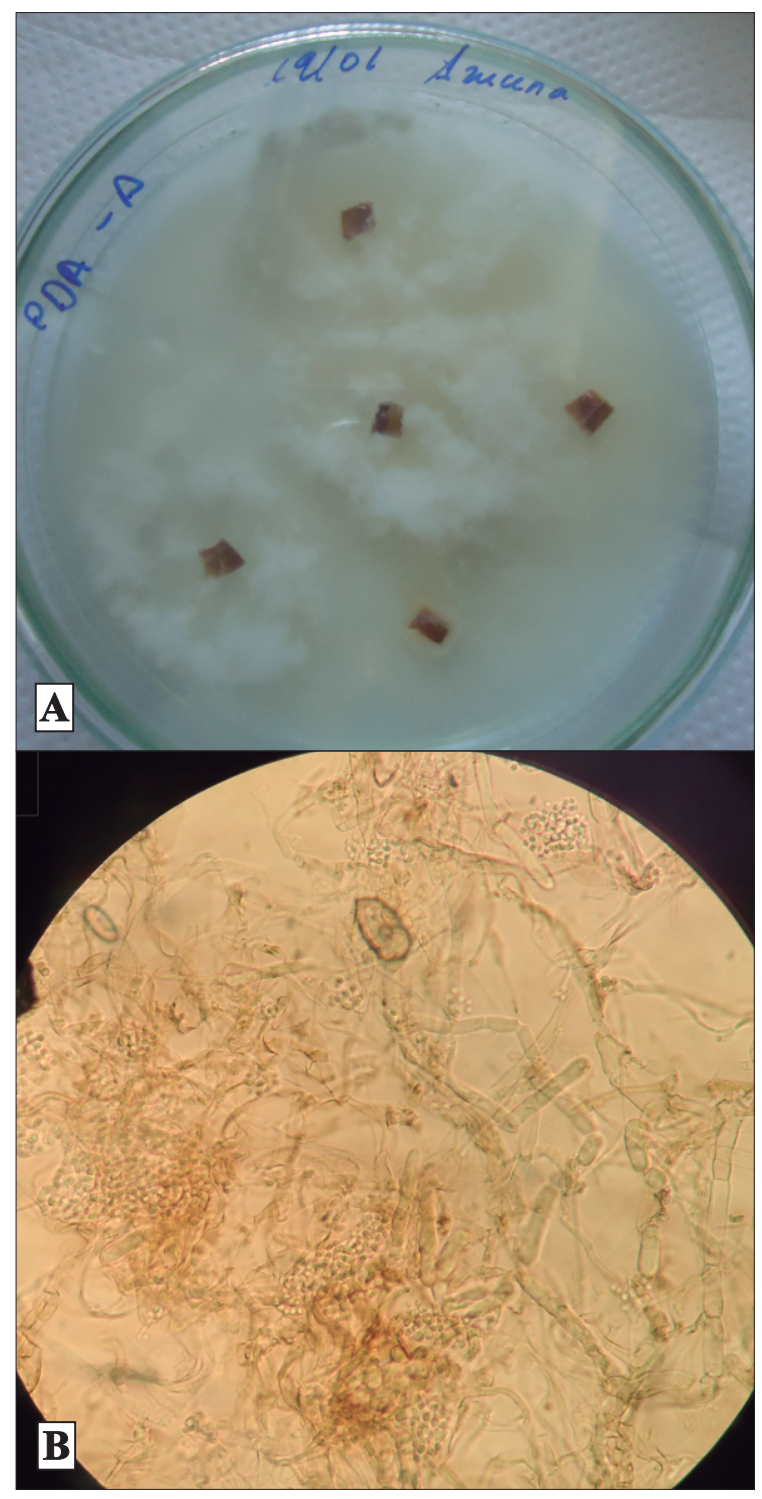

Figura 1. Colonias de Botrytis spp. aisladas de hojas de azucena (A), conidios en forma de racimo de Botrytis spp. en el microscopio a $63 \mathrm{x}(\mathrm{B})$.

Del mismo modo se logró aislar e identificar el agente causal de la antracnosis en los frutos de mango, en este caso Colletotrichum spp; ocho días después de la incubación, el crecimiento micelial llenó la placa Petri, el color del micelio fue anaranjado claro, y al observarse estas colonias en el microscopio se identificaron conidios cortos y erectos en forma de bala, morfología característica de éste género (Figura 2).

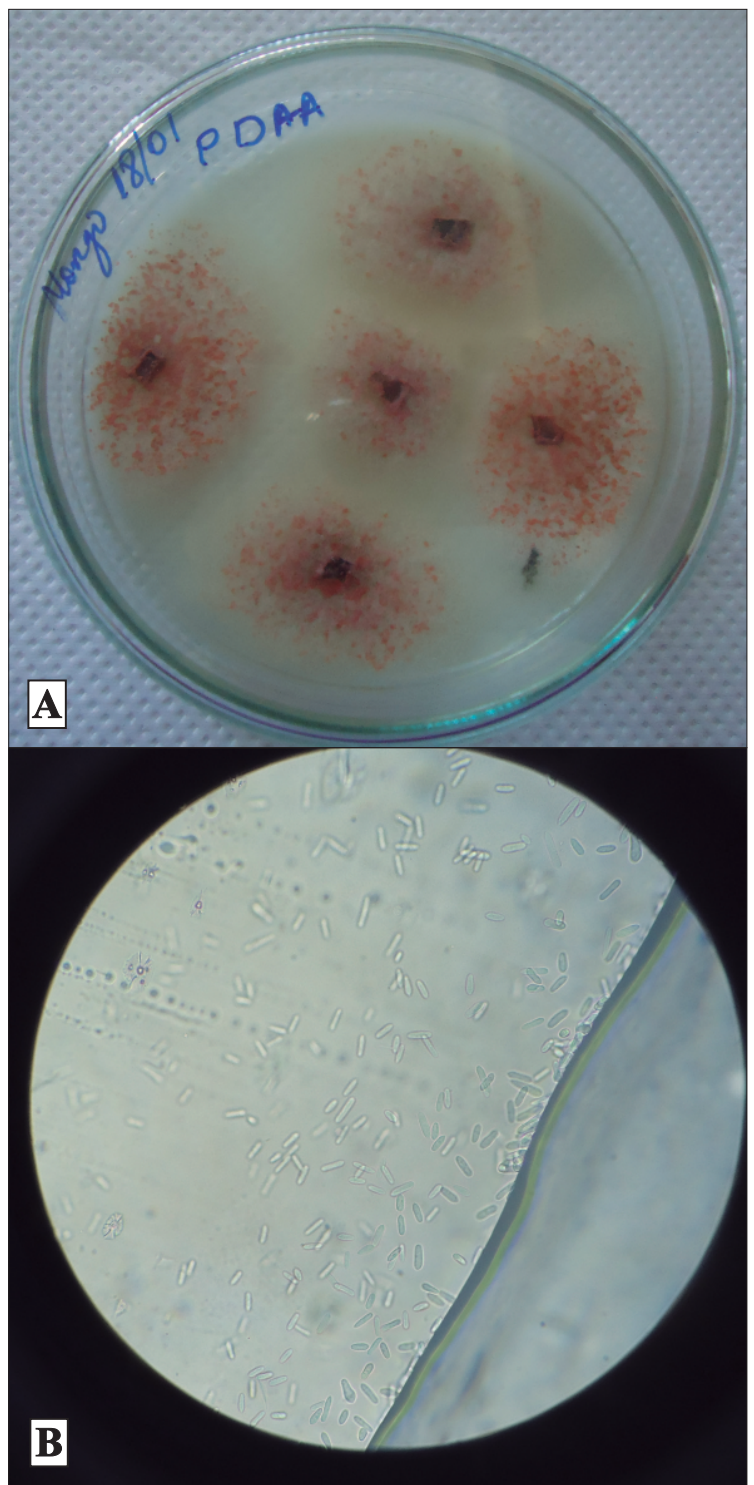

Figura 2. Colonias de Colletotrichum spp. aislado de frutos de mango (A), conidios cortos y erectos en forma de bala de Colletotrichum spp. vista en el microscopio a 63x (B).

\section{Comparación del crecimiento radial de Botrytis} spp. y Colletotrichum spp. enfrentados a los controladores biológicos

La figura 3 muestra el crecimiento micelial de Botrytis spp al día seis (144 horas) después del enfrentamiento. Los tratamientos T6 (testigo absoluto) y T4 (Clonostachys rosea), reportaron mayor crecimiento radial del patógeno $(4,0 \mathrm{~cm}$ y $3,7 \mathrm{~cm}$, respectivamente), que además presentan diferencias significativas estadísticas con los tratamientos T1 $(1,4 \mathrm{~cm}), \mathrm{T} 2(1,5 \mathrm{~cm}), \mathrm{T} 3$ 
$(1,4 \mathrm{~cm})$ y T5 $(2,5 \mathrm{~cm})$.

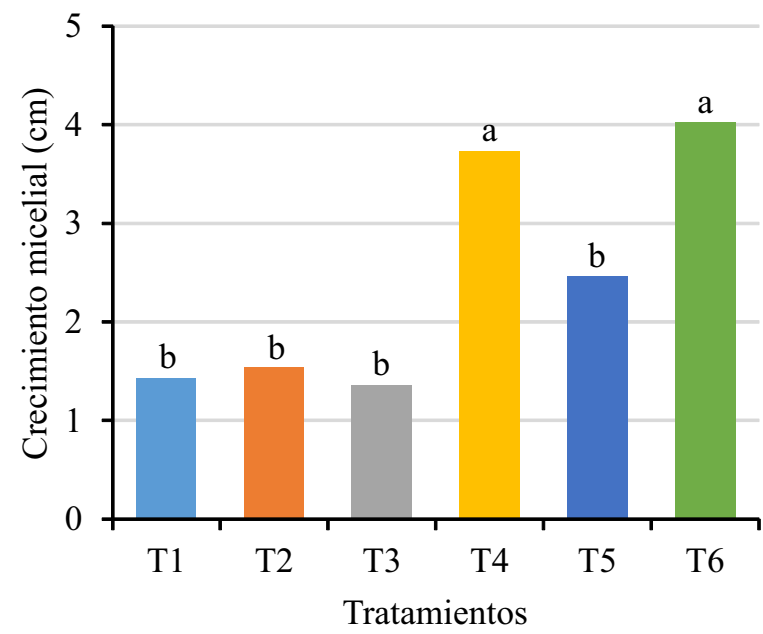

Figura 3. Crecimiento micelial en cm de Botrytis spp. al día seis (144 horas) después del enfrentamiento.

Los datos del crecimiento micelial de Colletotrichum spp. al día ocho (192 horas) después del enfrentamiento, se muestran en la figura 4, donde se puede observar que el tratamiento T6 presenta el mayor crecimiento micelial $(3,83 \mathrm{~cm})$, seguido del T4. Sin embargo, no se evidencian diferencias estadísticas significativas entre ellos. En el otro extremo está el tratamiento T3, que reporta el menor crecimiento micelial $(1,15 \mathrm{~cm})$.

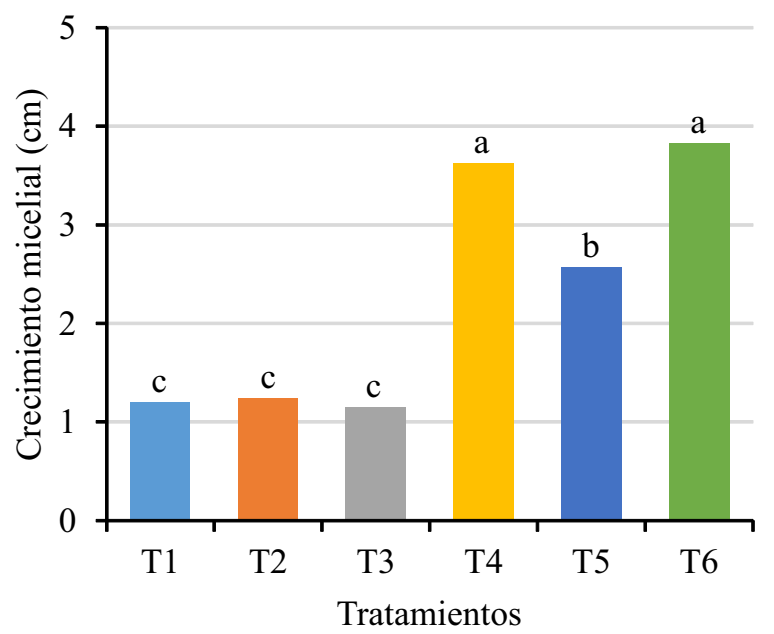

Figura 4. Crecimiento micelial de Colletotruchum spp al día ocho (192 horas) después del enfrentamiento.

\section{Porcentaje de inhibición del crecimiento radial}

El porcentaje de inhibición del crecimiento radial de Botrytis spp. se muestra en la figura 5, en la cual se puede observar que con el tratamiento T3 (T. asperellum) presenta el mayor porcentaje de inhibición
$(65,6 \%)$. De nuevo, no presenta diferencias significativas estadísticas con los tratamientos T1 $(64,2 \%)$ y T2 (61,8\%). Por otro lado, el T5 (testigo químico) exhibe el menor porcentaje de inhibición del crecimiento radial $(38,2 \%)$, seguido del $\mathrm{T} 4(6,3 \%)$.

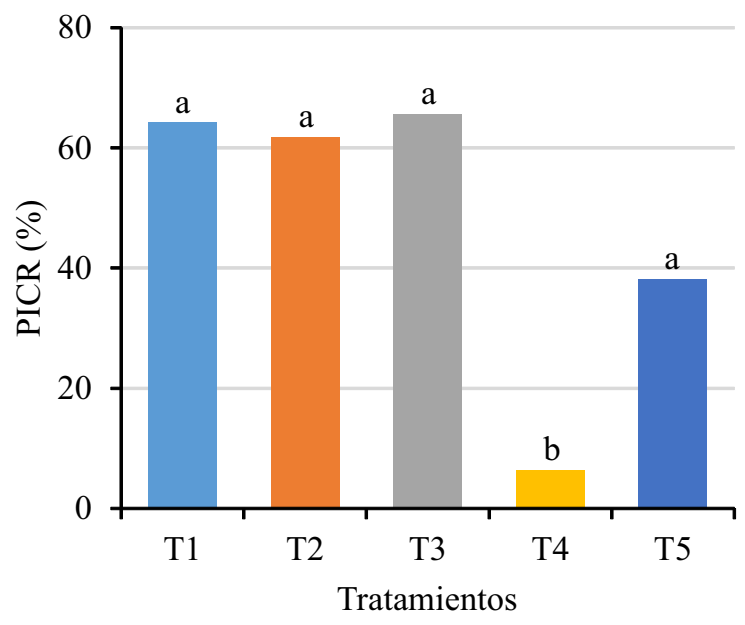

Figura 5. Efecto de los controladores biológicos en el porcentaje de inhibición del crecimiento radial de Botrytis spp.al día seis después del enfrentamiento.

El porcentaje de inhibición del crecimiento radial de Colletotrichum spp. se muestra en la figura 6, donde se puede observar que el tratamiento T3 (T. asperellum) presenta el mayor porcentaje de inhibición $(69,4 \%)$, sin presentar diferencias significativas estadísticas con los tratamientos T1 (68,7\%) y T2 (67,1\%). A su vez, el T5 (testigo químico) exhibe el menor porcentaje de inhibición del crecimiento radial $(28,1 \%)$, seguido del T4 (18,2\%), con el cual no se evidenciaron diferencias estadísticas significativas.

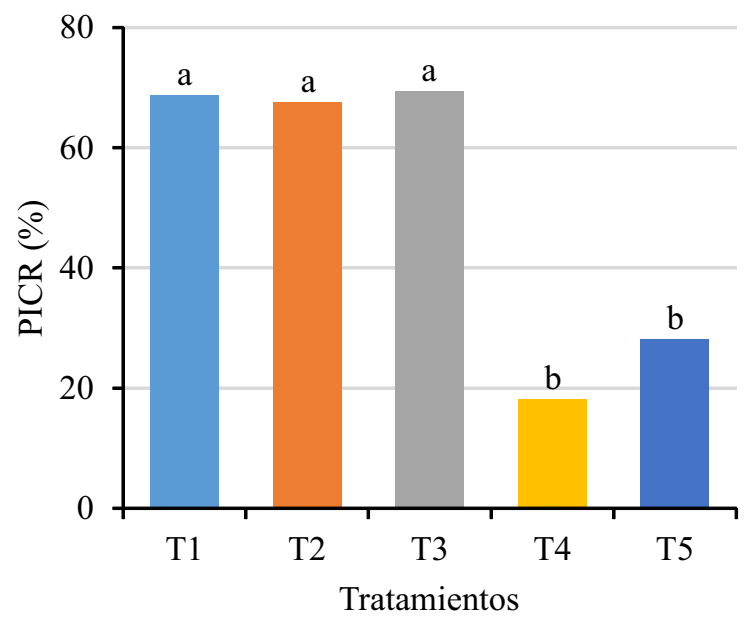

Figura 6. Efecto de los controladores biológicos en el porcentaje de inhibición del crecimiento radial de Colletotrichum spp. al día ocho después del enfrentamiento. 


\section{IV.DISCUSIÓN}

El mayor crecimiento micelial de los patógenos en el testigo absoluto es debido a la ausencia de controladores, ya que en los tratamientos que se enfrentó a un controlador, el crecimiento micelial del patógeno fue menor, sin embargo, tanto para Colletotrichum spp. como para Botrytis spp., de los cuatro controladores biológicos a los que fueron enfrentado, las cepas del género Trichoderma son las que presentaron menor crecimiento de los patógenos, diferenciándose significativamente $(\mathrm{p} \leq 0,5)$ de los tratamientos con $C$. rosea.

En el caso del porcentaje de inhibición del crecimiento radial, las cepas de Trichoderma alcanzaron los mayores porcentajes de inhibición sobre Colletotrichum y Botrytis spp., que oscilaron entre 65,6-61,8\% y 96,4\%-67,2\% respectivamente. Resultados similares obtuvieron Astorga-Quirós et al. (2013) al evaluar la capacidad antagónica de cepas de Trichoderma spp. sobre Penicilium spp. estos mostraron que las cepas de Trichoderma inhibieron el crecimiento radial en un 49\%. Así mismo Calvo-Araya et al. (2012), al evaluar in vitro diversos aislamientos de antagonistas sobre Botrytis cinerea, encontraron que las cepas de Trichoderma spp. presentaron los valores más altos de crecimiento radial con respecto al patógeno, así como un porcentaje de inhibición de B. cinerea superior al 90\%, mostrando que pueden ser potencial de control biológico para este patógeno.

En un estudio realizado por Ghosh y Chakraborty (2012), al estudiar el control biológico de Colletotrichum gloesporioides, llegaron a la conclusión que las cepas de Trichoderma viridae son buenos agentes de control, ya que sus esporas se localizaron adheridas en la pared y la contracción de protoplasto de C. gloeosporioides, ocasionando malformaciones e hinchamiento.

Por otro lado la cepa de C. rosea alcanzó un PICR de 6,3 a 18,2\% para Botrytis spp y Colletotrichum sp, respectivamente. Resultados similares obtuvieron Flores et al. (2015) al evaluar el porcentaje de inhibición de crecimiento radial de C. rosea sobre Botrytis cinerea, Alternaria solani y Fusarium Oxisporum, donde encontraron que los PICR fueron de 11,3\%, $1,5 \%$ y $9,9 \%$, respectivamente; sin embargo, los estudios con C. rosea son limitados y poco conocidos (Costa et al,. 2013).

\section{CONCLUSIONES}

Se logró identificar al agente causal de la antracnosis de los frutos de mango (Colletotricum spp.) y el agente causal de la rancha en azucena (Botrytis spp.).

El crecimiento micelial de Botrytis spp. y Colletotricum spp. fue menor que el de los testigos al ser enfrentados con las cepas de Trichoderma spp., con mayores porcentajes de inhibición del crecimiento radial de los patógenos, lo que significa que pueden ser una buena alternativa de control biológico para estos dos patógenos, y de esta manera, disminuir el uso de productos químicos, que son los principales causantes de la contaminación ambiental y además, perjudican la salud humana.

\section{REFERENCIAS BIBLIOGRÁFICAS}

Anderson, P.K., A.A. Cunningham, N.G. Patel, F.J. Morales, P.R. Epstein, y P. Daszak. "Emerging infectious diseases of plants: pathogen pollution, climate change and agrotechnology drivers". Trends Ecol. Evol., Vol. 19(2004): 535-544.

Archie, E.A., G. Luikart, y V.O. Ezenwa. "Infecting epidemiology with genetics: a new frontier in disease ecology". Trends Ecol. Evol., Vol. 24(2009): 21-30.

Astorga-Quirós, K, K. Meneses-Montero, C. ZúñigaVega, J. Brenes-Madriz, y W. Rivera-Méndez. "Evaluación del antagonismo de Trichoderma sp. y Bacillus subtilis contra tres patógenos del ajo". Tecnología en Marcha, Vol. 27 (2013): 82-91.

Calvo-Araya, J.A., G. Rivera-Coto, S. OrozcoCayasso, y R. Orozco-Rodríguez. “Aislamiento y evaluación in vitro de la antagonistas de Botrytis cinerea en mora". Agron. Mesoam, Vol. 23(2012): 225-231. 
Costa, L.B., D.E. Rangel, M.A. Morandi, y W. Bettiol. "Effects of UV-B radiation on the antagonistic ability of Clonostachys rosea to Botrytis cinerea on strawberry leaves". Biological Control, Vol. 65(2013): 95-100.

Farooq, M., K. Jabran, Z. Cheema, A. Wahid, y K.H. Siddique. "The role of allelopathy in agricultural pest management". Pest management science, Vol. 67(2011): 493-506.

Fischbein, D. "Introducción a la teoría del control Introducción a la teoría del control". Buenos Aires (Argentina), 2012.

Flores, W., J. Chico, y L. Cerna. “Actividad antagónica in vitro de Clonostachys rosea sobre Fusarium oxysporum, Alternaria solani y Botrytis cinérea”. Revista REBIOL, Vol. 35(2015): 3442.

Ghosh, S.K., y N. Chakraborty. "In vitro biological control of Colletotrichum gloeosporioides, causal organism of anthracnose of sarpagandha (Roulvolfia serpentina)". Agricultutal Biological Journal of North América, Vol. 3(2012): 306-310.

Guédeza, C., L. Cañizáleza, C. Castilloa, y R. Olivarb. "Efecto antagónico de Trichoderma harzianum sobre algunos hongos patógenos postcosecha de la fresa (Fragaria spp)". Revista de la Sociedad Venezolana de Microbiología, Vol. 29(2009): 34-38.

Hernández, M., M. Murace, J. Ringuelet, I. Petri, D. Gallo, y A. Arambarri. "Effect of aqueous and alcohol extracts of Phytolacca tetramera (Phytolaccaceae) leaves on Colletotrichum gloeosporioides (Ascomycota)". Boletín de la Sociedad Argentina de Botánica, Vol. 48(2013): 201-209.

Infante, D., B. Martínez, N. González y Y. Reyes. "Mecanismos de acción de Trichoderma frente a hongos fitopatógenos". Revista de protección vegetal, Vol. 24(2009): 14-21.

Krugg, J.W., y H. Requejo-Valdivieso. “Aislamiento dehongosfitopatógenos en lesiones de hoja y tallo de cultivos de vid, Vitis vitícola, de Cascas, La Libertad (Perú)". Revista REBIOL, Vol. 32(2012): 56-51.

Leyva-Mir, S.G., Y. López-Hernández, B. TlapalBolaños, y R. Flores-Martínez. "Etiología del tizón descendente de las ramas de azucena híbrida (Lilium spp.) en Villa Guerrero, Estado de México". Revista Chapingo. Serie horticultura, Vol. 15(2009): 5-8.

Santander, A.J. “Uso de Trichoderma harzianun Rifai y Bacillus subtilis (Ehrenberg) Cohn para el control de (Colletotrichum gloeosporioides Pens.) causante de la antracnosis en mango (Mangifera indica L.)". Maracay (Venezuela), 2012.

Ryu, H., H. Park, D.S. Suh, G.H. Jung, K. Park, y B.D. Lee. "Biological control of Colletotrichum panacicola on Panax ginseng by Bacillus subtilis HK-CSM-1". Journal of ginseng research, Vol. 38(2014): 215-219. 